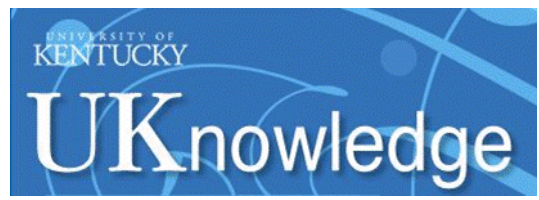

University of Kentucky

UKnowledge

\title{
On the Control of a Solid State Transformer for Multi-MW Utility- Scale PV-Battery Systems
}

Yibin Zhang

University of Kentucky, yibin.zhang@uky.edu

Oluwaseun M. Akeyo

University of Kentucky, ochichikeyo@gmail.com

Jiangbiao He

University of Kentucky, Jiangbiao.He@uky.edu

Dan M. lonel

University of Kentucky, dan.ionel@uky.edu

Follow this and additional works at: https://uknowledge.uky.edu/peik_facpub

Part of the Power and Energy Commons

Right click to open a feedback form in a new tab to let us know how this document benefits you.

\section{Repository Citation}

Zhang, Yibin; Akeyo, Oluwaseun M.; He, Jiangbiao; and Ionel, Dan M., "On the Control of a Solid State Transformer for Multi-MW Utility-Scale PV-Battery Systems" (2019). Power and Energy Institute of Kentucky Faculty Publications. 14.

https://uknowledge.uky.edu/peik_facpub/14

This Conference Proceeding is brought to you for free and open access by the Power and Energy Institute of Kentucky at UKnowledge. It has been accepted for inclusion in Power and Energy Institute of Kentucky Faculty Publications by an authorized administrator of UKnowledge. For more information, please contact UKnowledge@lsv.uky.edu. 


\title{
On the Control of a Solid State Transformer for Multi-MW Utility-Scale PV-Battery Systems
}

\author{
Digital Object Identifier (DOI) \\ https://doi.org/10.1109/ECCE.2019.8912477
}

\section{Notes/Citation Information}

Published in 2019 IEEE Energy Conversion Congress and Exposition (ECCE).

(C) 2019 IEEE Copyright Notice. "Personal use of this material is permitted. Permission from IEEE must be obtained for all other uses, in any current or future media, including reprinting/republishing this material for advertising or promotional purposes, creating new collective works, for resale or redistribution to servers or lists, or reuse of any copyrighted component of this work in other works."

The document available for download is the authors' manuscript version that is accepted for publication. The final published version is copyrighted by IEEE and is available as: Y. Zhang, O. Akeyo, J. He, and D. M. Ionel," On the Control of a Solid State Transformer for Multi-MW Utility-Scale PV-Battery Systems," 2019 IEEE Energy Conversion Congress and Expo (ECCE), Baltimore, MD, 2019, pp. 6481-6486, doi: 10.1109/ ECCE.2019.8912477 


\section{On the Control of a Solid State Transformer for Multi-MW Utility-Scale PV-Battery Systems}

\author{
Yibin Zhang \\ SPARK Lab, ECE Dept. \\ University of Kentucky \\ Lexington, KY, USA \\ yibin.zhang@uky.edu
}

\author{
Oluwaseun Akeyo \\ SPARK Lab, ECE Dept. \\ University of Kentucky \\ Lexington, KY, USA \\ m.akeyo@uky.edu
}

\author{
Jiangbiao He \\ AMPERE Lab, ECE Dept. \\ University of Kentucky \\ Lexington, KY, USA \\ jiangbiao.he@uky.edu
}

\author{
Dan M. Ionel \\ SPARK Lab, ECE Dept. \\ University of Kentucky \\ Lexington, KY, USA \\ dan.ionel@uky.edu
}

\begin{abstract}
The utility-scale photovoltaic (PV)-battery systems typically include multiple power converters connected to the grid via traditional line frequency transformers (LFT), which may be considered bulky, and inefficient when compared with the emerging solid state transformers (SST). This paper proposes a SST with power and voltage controls for utility-scale PVbattery systems. The PV system was modeled based on available data from a universal solar facility located in Kentucky, USA. Furthermore, this paper provides detailed controller designs for the proposed utility-scale PV-battery system based on a SST which includes: the PV-side dual-active bridge (DAB) converter tracks the maximum power point, the $\mathrm{PV}$ inverter for steady power transformation to the grid, battery-side DAB converter for maintaining stiff dc bus voltage, and the battery inverter for voltage support and balanceing the power mismatch between the grid demand and the PV generation. Moreover, medium frequency transformer (MFT) and Silicon Carbide (SiC) MOSFETs are utilized in the DAB converters and inverters, to improve the efficiency of the PV-battery system. The simulation results based on the data retrieved from an operational PV facility, on one hand, are presented to confirm the benefits of the proposed $\mathrm{SiC}$ SST based PV-battery system; on the other hand, provide an alternative analysis on power losses and efficiency for the utilityscale PV farms.
\end{abstract}

Index Terms-Solid state transformer, voltage control, power gap balancing, Silicon Carbide, PV farm applications.

\section{INTRODUCTION}

Over the past decade, photovoltaic (PV) power generation has experienced rapid development due to its numerous advantages [1]. Among various PV applications, the utility-scale $\mathrm{PV}$ farm is able to provide power grid with a large capacity of solar energy. Due to solar intermittency nature, energy storage systems such as battery energy storage (BES) may need to be locally installed to provide ancillary services and enhanced stability for PV integration to power grid [2].

The traditional grid-connected PV-BES system (Fig. 1) consists of line frequency transformers (LFT) and power inverters, while some systems also contain $\mathrm{dc} / \mathrm{dc}$ converters for achieving the algorithm of maximum power point tracking (MPPT). However, the traditional LFT is inefficient, bulky, and may cause additional harmonics at high load percentage [3]. Every time BES provides services such as power smoothing, voltage sag regulation, and power gap balancing, the LFT for BES system lower the overall system efficiency. The situation might become worse when PV-BES feeds local loads through several more distribution LFTs.

As a smarter transformer, solid state transformer (SST) possesses decreased volume and weight, and higher power density, while potentially offering many smart features such as voltage regulation, fault isolation and bidirectional power flow [4]. Much effort has been made on SST and four power conversion topologies have been proposed in which the medium frequency transformers (MFT) are utilized [5]. These topologies include single-stage, two-stage with low-voltage-dc link, two-stage with medium-voltage-dc link, and three-stage with low-voltage- and medium-voltage-dc link shown in Fig. 2 [6]. Among these topologies, two-stage with medium-voltagedc link has higher power density, while three-stage SST can achieve most of the smart features but with lower efficiency.

With the aforementioned merits of SST, researchers have been developing its applications for medium- and utility-scale PV systems [7][8]. The MFTs are widely designed to improve the power density and efficiency [9][10]. Besides, the voltage controller is presented [11] to calculate the duty cycle based on dc link and reference voltages. A detailed voltage control scheme with efficiency improvement is developed in [12]. However, the control for renewable generation systems requires a more sophisticated scheme including the coordination with battery energy storage (BES) and other ancillary services such as peak-shaving valley-filling.

In this paper, the main contributions can be summarized as follows. 1) A SST is chosen here considering the smart function implementation and efficiency improvement; 2) PV and BES controllers are designed. The MPPT, dc-link voltage control, and grid synchronization are achieved with the proposed PV controller. For the BES controller, the power gap between PV farms and load is balanced, and the dc-link voltage of $\mathrm{BES}$ is controlled; 3) $\mathrm{SiC}$ semiconductor devices are selected for the 10MW PV farm, to reduce power losses. The power loss and efficiency analysis are investigated.

The detailed modeling and controller designs of the proposed SST for 10MW PV-battery systems will be introduced in Section II. Furthermore, power device specifications, power and voltage regulation for solar partial shading, power loss and efficiency investigation will be presented in Section III. Finally, conclusions will be given in Section IV. 


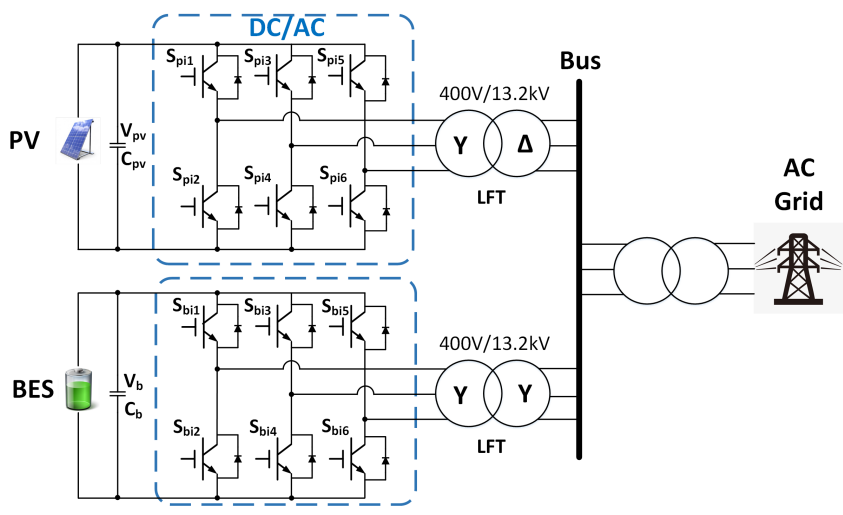

Fig. 1: The conventional power architecture of utility-scale PV-BES systems with inverters and LFTs.

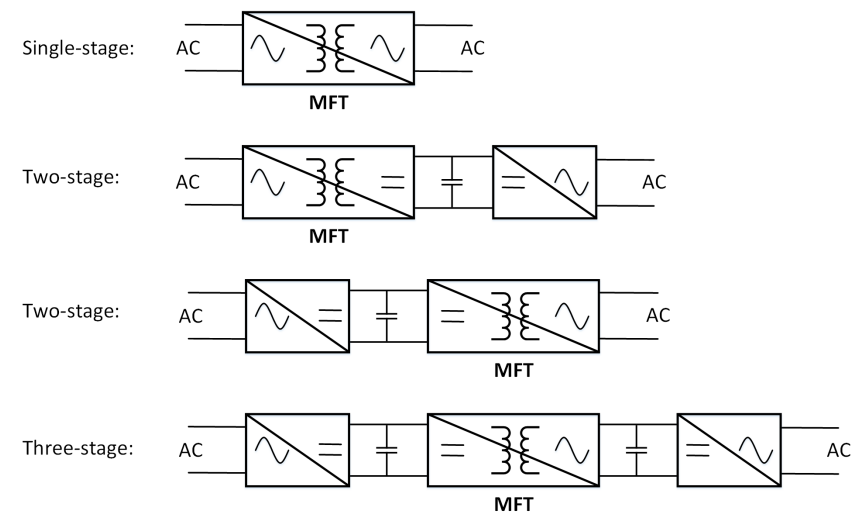

Fig. 2: The circuit topology classification of SST.

\section{SySTEM MODELING}

In this section, the detailed modeling and performance investigation of PV arrays, DAB converters, inverters, and controllers for both PV and BES will be described as developed for implementation in an electric circuit simulation software such as the ANSYS/TwinBuilder [14]. The experimental data for the study, in terms of solar irradiance and output power, is provided by a $10 \mathrm{MW}$ utility-scale PV farm operated by the LG\&E and Kentucky Utilities on the E.W. Brown site (Fig. $3 a)$, and such data is available online [15].

\section{A. PV Array Modeling}

The PV array was modeled based on an operational 10MW PV farms, which consists of multiple 1MW array sections. Each 1MW array section was modeled to connect to ac grid via one two-level inverter and one LFT. The simulation results of the PV power matches the recorded data of the facility (Fig. 3b). Furthermore, a SST with LVDC and MVDC links is designed for the 10MW PV-battery system (Fig. 4). In the proposed architecture, the PV panels are connected to the ac bus in 30 SST arrays. Each array which includes one DAB converter, one three-phase inverter, and one MFT, is rated for $35 \mathrm{~kW}$ due to the current manufacture of MFTs.

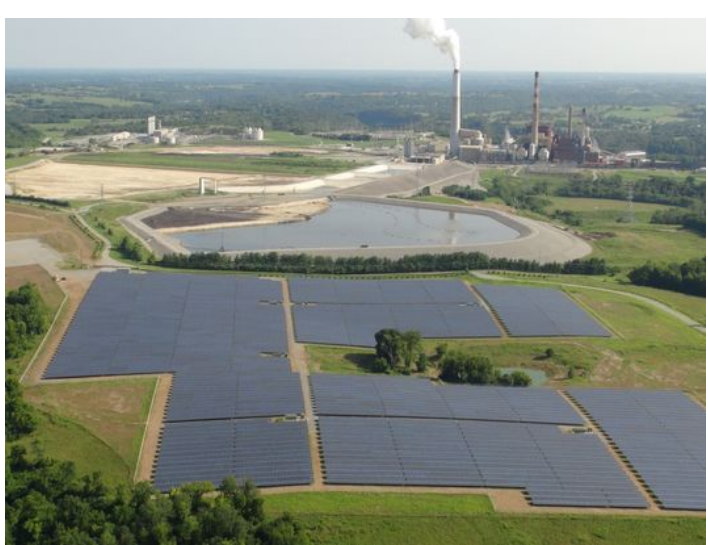

(a)

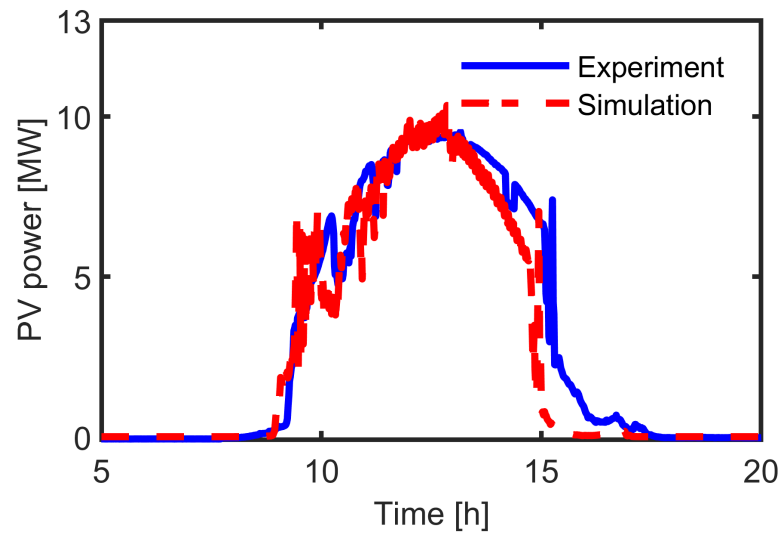

(b)

Fig. 3: The experimental data and validation in simulation, (a) arial view of the E. W. Brown 10MW PV farm in Kentucky, which provided the experimental data considered in the study [13], and (b) the PV power data in a winter day and the simulated power data.

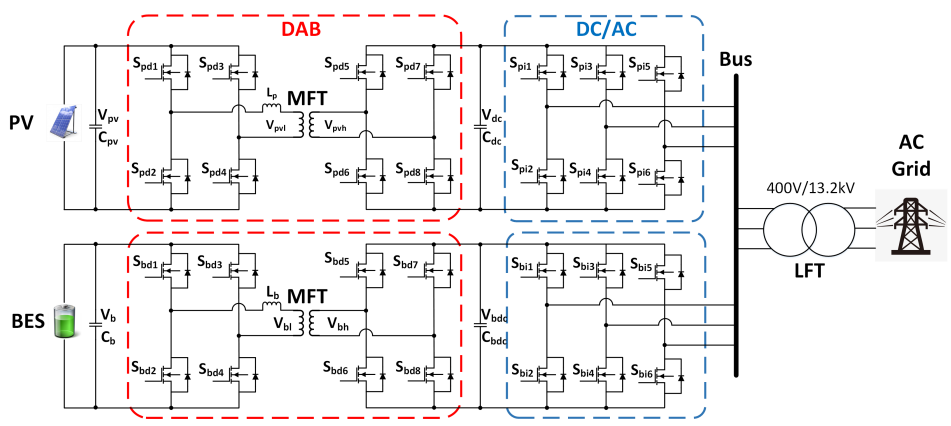

Fig. 4: The proposed SST based PV-BES system: PV arrays and BES are connected through their own set of DAB and inverter to ac bus. 


\section{B. DAB and Controller Modeling on PV-Side}

For each DAB based PV array, the output voltage in both $\mathrm{H}$-bridges can be expressed in Fourier series form as in Eq. (1) and Eq. (2). Also, the currents flowing through the DAB converter and the dc-bus voltage can be expressed in Eq. (3) and Eq. (4). Based on the aforementioned analysis, the active power with different series orders can be presented in Eq. (5).

$$
\begin{gathered}
v_{p v l}(t)=\sum_{n=1,3, . .}(-1)^{\frac{(n-1)}{2}} \frac{4 V_{p v l}}{n \pi} \sin \left(n \pi d_{p}\right) \\
v_{p v h}(t)=\sum_{n=1,3, . .}(-1)^{\frac{(n-1)}{2}} \frac{4 V_{p v h}}{n \pi} \sin \left(n \pi d_{s}\right) \\
i_{L}(t)=\frac{1}{L_{p}} \int\left[v_{p v l}(t)-\frac{1}{K} v_{p v h}(t)\right] d t \\
C_{d c} \frac{d}{d t} V_{d c}(t)=i_{p v h}-i_{i n v p} \\
P=\sum_{n=1,3, . .} \frac{8 V_{p v h} V_{p v l}}{K n^{3} \pi^{2} \Omega L_{p}} \sin \left(n \pi d_{s}\right) \sin \left(n \pi d_{p}\right)
\end{gathered}
$$

where $n$ refers to the order of Fourier series; $v_{p v l}$ and $v_{p v h}$ represent primary and secondary side voltages of the mediumfrequency transformer (HFT), respectively; $K, L_{p}$, and $C_{d c}$ stand for turns ratio of the HFT, inductance, and capacitance shown in Fig. 4, respectively.

To model the PV controllers shown in Fig. 5, firstly, a MPPT controller is employed to regulate the PV output voltage $V_{p v}$, which provides a constant dc voltage for the two sides of the HFT. Furthermore, the inverter follows the dc voltage and grid status to maintain grid connected and provide a constant power at the required voltage.

\section{DAB and Controller Modeling on BES-Side}

In the example configuration, the BES is connected to $13.2 \mathrm{kV}$ ac bus to regulate the imbalance and voltage sag (Fig. 6). However, in this scenario, every time BES provides voltage sag regulation, power smoothing, or power gap balancing, the power has to flow via two conventional LFTs which dominates one of the major losses in the power conversion system. For the local loads, it is even worse to pass power through several more distribution transformers.

In the proposed SST based system, the employed HFTs help further reduce the losses through transformers. The operating principle of the BES is similar to the process shown for the $\mathrm{DAB}$ and controller modeling of the PV-side. For the controller design, firstly, a voltage regulator is designed to extract a constant dc voltage for the inverter to follow. Then the power gap between the PV and load is considered to modulate the BES inverter. When the solar energy is sufficient, PV provides power to the grid and battery charging; when the solar is insufficient, BES starts to discharge to provide constant power to the load. In this scenario, the oscillation caused by the solar

\begin{tabular}{|c|c|c|}
\hline Items & Descriptions & Values \\
\hline$f$ & The system frequency & $60 \mathrm{~Hz}$ \\
\hline$P_{p v}$ & MPP power of PV & $10 \times 1 \mathrm{MW}$ \\
\hline$V_{p v}$ & dc link voltage of PV & $750 \mathrm{~V}$ \\
\hline \multirow[t]{4}{*}{$V_{\text {bus }}$} & The RMS value of ac bus voltage & $13.2 \mathrm{kV}$ \\
\hline & The number of PV arrays & 10 \\
\hline & Series PV module in one string & 19 \\
\hline & Parallel Strings & 168 \\
\hline$f_{s}$ & The switching frequency & $5 \mathrm{kHz}$ \\
\hline$P_{b}$ & The power of BES & $2 \mathrm{MWh}$ \\
\hline$V_{b}$ & The output voltage of BES & $200 \mathrm{~V}$ \\
\hline$L_{p}$ & The DAB inductor & $30 \mathrm{mH}$ \\
\hline$C_{p v}$ & The input capacitor & $22 \mathrm{mF}$ \\
\hline$C_{d c}$ & The output capacitor & $18.15 \mathrm{mF}$ \\
\hline$f_{\text {sic }}$ & The switching frequency of SiC MOSFETs & $10 \mathrm{kHz}$ \\
\hline$f_{s i}$ & The switching frequency of Si IGBTs & $5 \mathrm{kHz}$ \\
\hline$k_{p}$ & $\begin{array}{l}\text { The proportional gain of } \mathrm{PV} \text { and BES con- } \\
\text { trollers }\end{array}$ & 0.00167595 \\
\hline$k_{i}$ & The integral gain of PV and BES controllers & 0.11173 \\
\hline$P_{m f t}$ & The power rating of MFT & $35 \mathrm{kVA}$ \\
\hline$f_{m f t}$ & The operating frequency of MFT & $5 \mathrm{kHz}$ \\
\hline$K_{m f t}$ & The turns-ratio of MFT & $32 / 32$ \\
\hline$R_{m f t 1}$ & The winding resistance of MFT primary side & $6.25 \Omega$ \\
\hline$R_{m f t 2}$ & The winding resistance of MFT secondary side & $0.1882 \Omega$ \\
\hline$L_{m f t d 1}$ & The leakage inductance of MFT primary side & $6.1 \mathrm{uH}$ \\
\hline$L_{m f t d 2}$ & $\begin{array}{l}\text { The leakage inductance of MFT secondary } \\
\text { side }\end{array}$ & $24.4 \mathrm{uH}$ \\
\hline$L_{m f t m}$ & The magnetization inductance of MFT & $67.64 \mathrm{mH}$ \\
\hline$K_{l f t}$ & The voltage ratio of LFT & $400 / 13200$ \\
\hline$P_{l f t}$ & The power rating of LFT & 1MVA \\
\hline$R_{l f t 1}$ & The winding resistance of LFT primary side & $0.00228 \Omega$ \\
\hline$R_{l f t 2}$ & The winding resistance of LFT secondary side & $6.25 \Omega$ \\
\hline$L_{l f t d 1}$ & The leakage inductance of LFT primary side & $0.01613 \mathrm{H}$ \\
\hline$L_{l f t d 2}$ & The leakage inductance of LFT secondary side & $0.49736 \mathrm{H}$ \\
\hline
\end{tabular}
intermittent or partial shading, can be avoided by the proposed BES controller, which enhances the stability and reliability of the grid-connected PV system.
TABLE I: The system parameters

\section{Simulation AND EXPERIMENTAL RESUlTS}

In this section, the proposed SST based PV-BES system and the designed controllers was simulated based on the $10 \mathrm{MW}$ PV experimental data. The parameters of the system configurations are listed in Table I. Furthermore, power losses and overall efficiency will be taken into consideration. The benefits of SiC based SST PV-BES system over the conventional Si systems was analyzed.

\section{A. Voltage Sag Compensation}

A scenario of solar partial shading is studied based on the irradiance data in a winter day [15]. In the beginning, the irradiance is sufficient at $1000 \mathrm{~W} / \mathrm{m}^{2}$. The PV panels feed $9.8 \mathrm{MW}$ with the MPPT strategy implemented at $0.8 \mathrm{kV}$ (Fig. 7). During this period, there is no operation from the BES, and PV farms contribute only real power to grid/loads with $i_{q}=$ 0 and $i_{d}=1 \mathrm{~A}$ as shown in Fig. 8. At $25 \mathrm{~s}$, the partial shading happens and the irradiance drops to $400 \mathrm{~W} / \mathrm{m}^{2}$ and lasts for $35 \mathrm{~s}$ of duration. The PV panels are only able to supply $3.8 \mathrm{MW}$ at $0.73 \mathrm{kV}$ to dc link which cannot meet the requirement from the load. The BES starts to discharge $2 \mathrm{MW}$ and provides voltage support (Fig. 9). Therefore, the designed BES controller balances the power gap and compensates the voltage sag. During the solar partial shading, no voltage drop, oscillation or distortion occurs on the output voltage. Accordingly, the proposed SST based PV-BES system provides synchronized and stable power supply to the local load following the grid. 


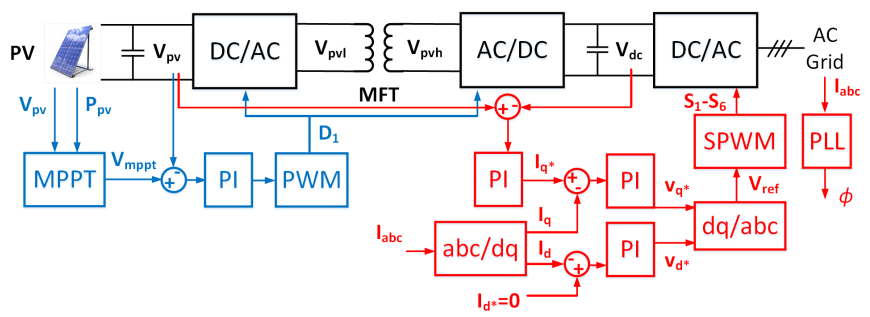

Fig. 5: The PV controller diagram with MPPT and gridsynchronization.

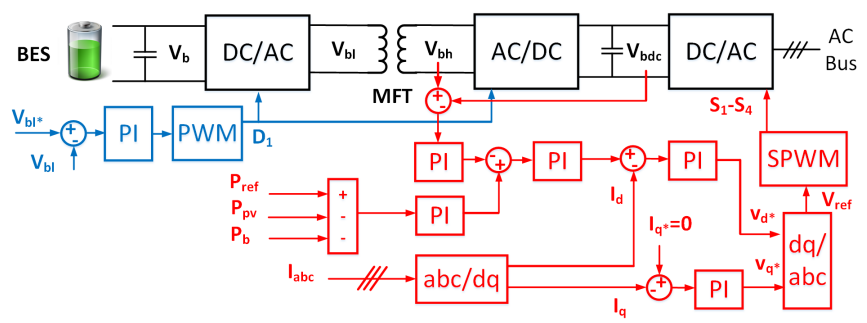

Fig. 6: The BES controller diagram with voltage sag compensation and power gap balancing.

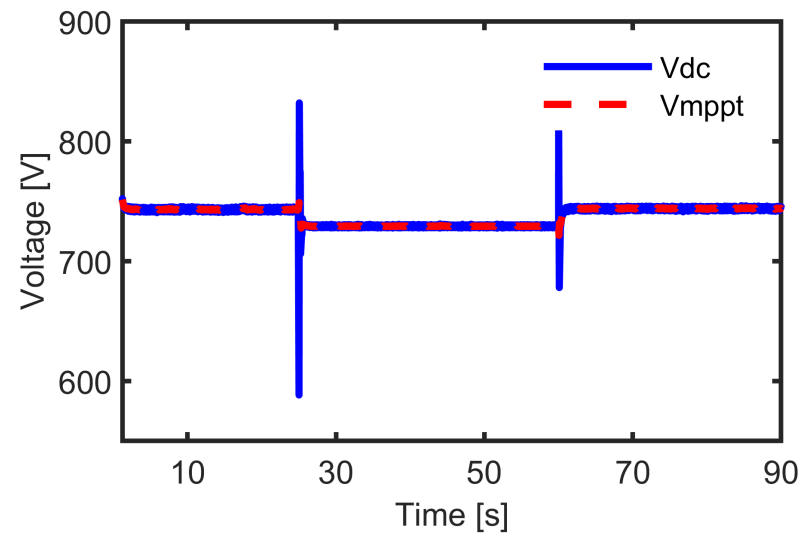

Fig. 7: The voltage results of MPPT voltage and dc voltage in the case of solar partial shading during $25 \mathrm{~s}-60 \mathrm{~s}$.

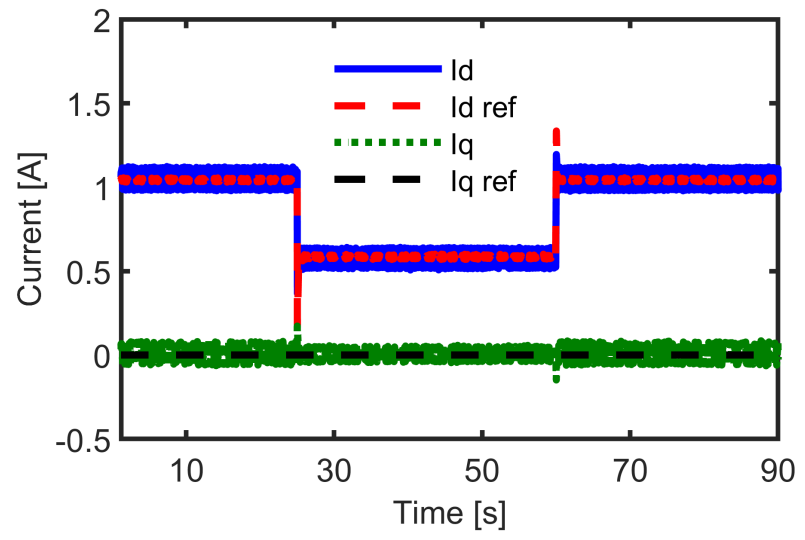

Fig. 8: The current results in the case of solar partial shading during 25s - 60s: Id, Iq, and reference currents.

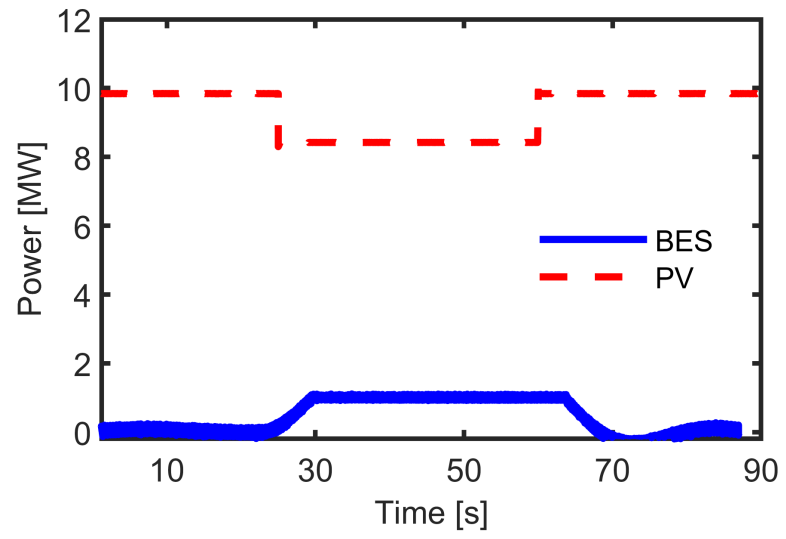

Fig. 9: Simulation results of PV output power and BES output power in the case of solar partial shading during $25 \mathrm{~s}-60 \mathrm{~s}$.

\section{B. Power Loss Investigation on Semiconductor Devices}

Since the PV farm is connected to grid with multiple arrays, in this section, losses on each $200 \mathrm{~kW}$ array is investigated for the mode of PV feeding load/grid. For the conventional PV system, Infineon Si IGBT module FF1200R12KE3 [16] rated at $1200 \mathrm{~V} / 1200 \mathrm{~A}$ is selected as an example for the twolevel three-phase inverters. For each array, the PV array and its inverter is operated at $5 \mathrm{kHz}$ and rated $200 \mathrm{~kW}$. For the SiC based SST PV-BES system, Wolfspeed SiC MOSFET module CAS300M12BM2 [17] (rated at 1200V/300A and operated at $10 \mathrm{kHz}$ switching frequency) is used for this study of converters to configure $1000 \mathrm{~A}$ at $200 \mathrm{~kW}$. Therefore, in the proposed system, the number of power semiconductor devices is increased, and the total conduction loss is increased accordingly. At nominal load condition, the conduction loss of the switch $s_{p i 1}$ (Fig. 10) in the conventional PV system is $68 \mathrm{~W}$, which is $34 \mathrm{~W}$ lower than the proposed SST system $(6 \times 17 \mathrm{~W}$ for one $200 \mathrm{~kW}$ array in Fig. 11).

Including semiconductor switching losses and diode recovery losses, the total switching loss of the proposed SST based system is significantly reduced, even though the switching frequency is twice as in the Si IGBT inverter system. At nominal load condition, the conduction loss of the switch $s_{p i 1}$ in the conventional system is $443 \mathrm{~W}$, which is $430.4 \mathrm{~W}$ higher than the proposed SST system $(6 \times 2.1 \mathrm{~W}$ for one $200 \mathrm{~kW}$ array in Fig. 13).

In conclusion, the total power losses on the power semiconductor devices under different load percentage can be summarized and compared for the 1MW PV farm (Fig. 14). It can be seen that total power loss in the proposed $\mathrm{SiC}$ SST system is reduced for each 1MW array. And in the next section, the modeling and loss analysis of transformers will be discussed and the overall system efficiency can be provided.

\section{Investigation on Transformer and System Efficiency}

In this section, the in-use LFT 1.25MVA transformer (Eaton Cooper Power Series 00001XA66X6JS, 1250kVA, 390Y is selected as an example for this study) [18] and the MFT [19] 


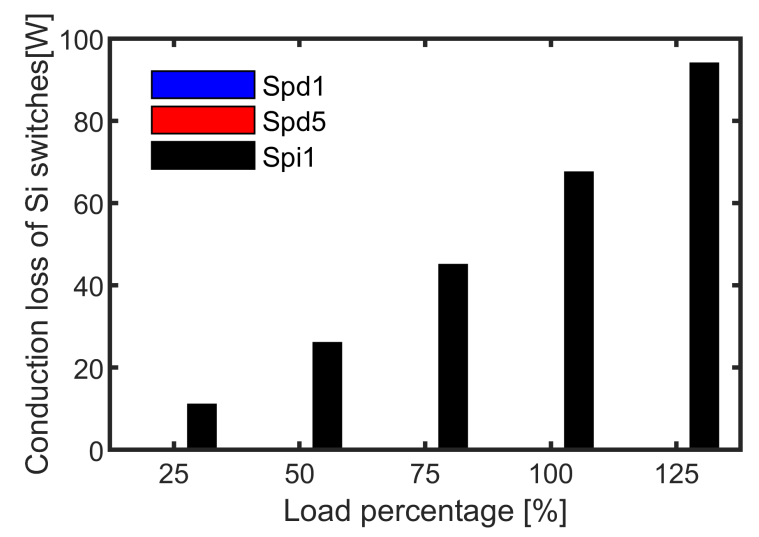

Fig. 10: The conduction losses of the conventional Si PV-BES system under different load percentage.

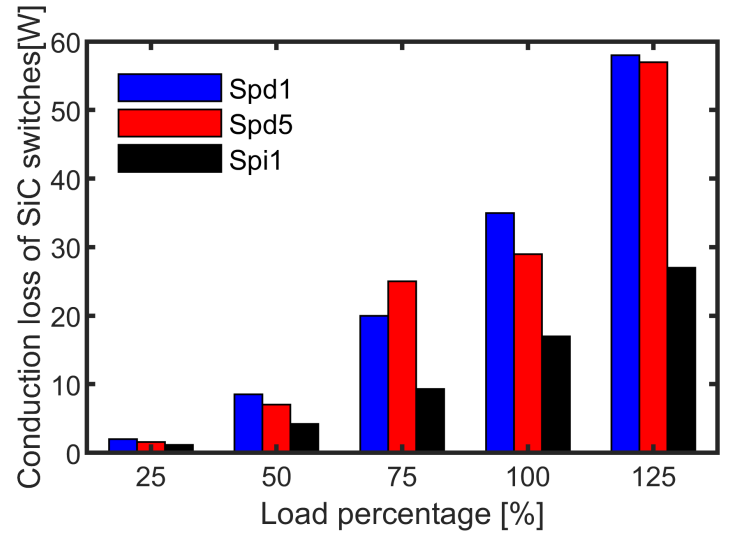

Fig. 11: The conduction losses of the proposed SiC based SST PVBES system under different load percentage.

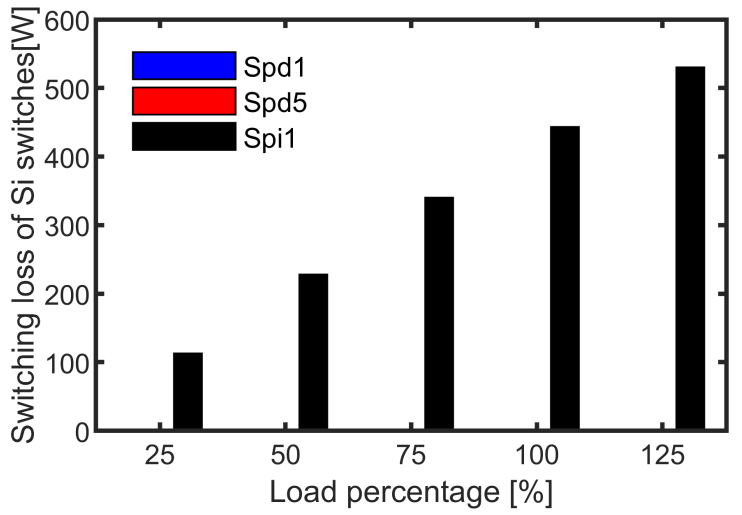

Fig. 12: The switching losses of the conventional Si PV-BES system under different load percentage.

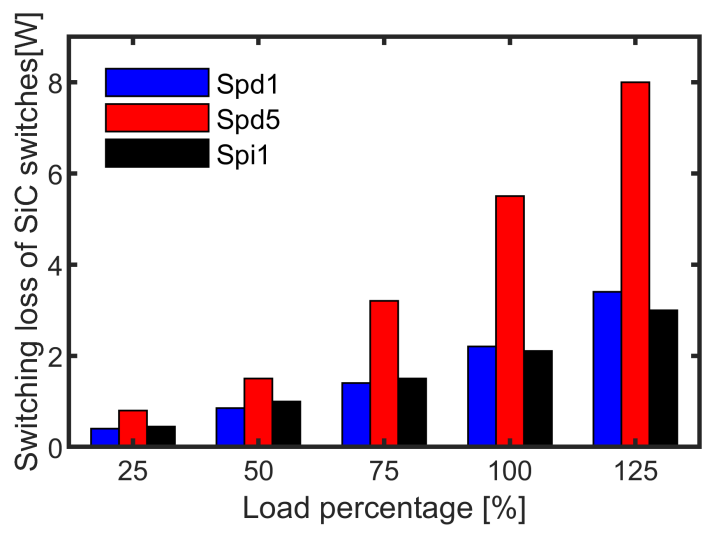

Fig. 13: The switching losses of the proposed SiC based SST PV-BES system under different load percentage.

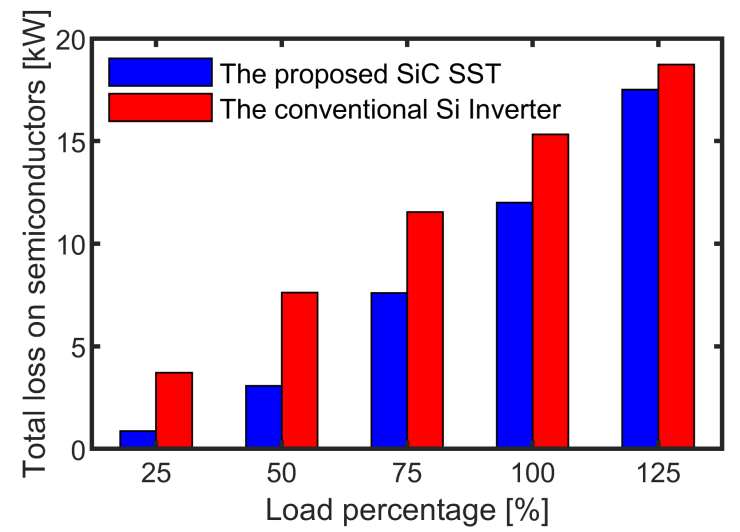

Fig. 14: The power loss comparison on power semiconductor switches for 1MW array between the proposed SiC SST based system and the conventional Si inverter system.

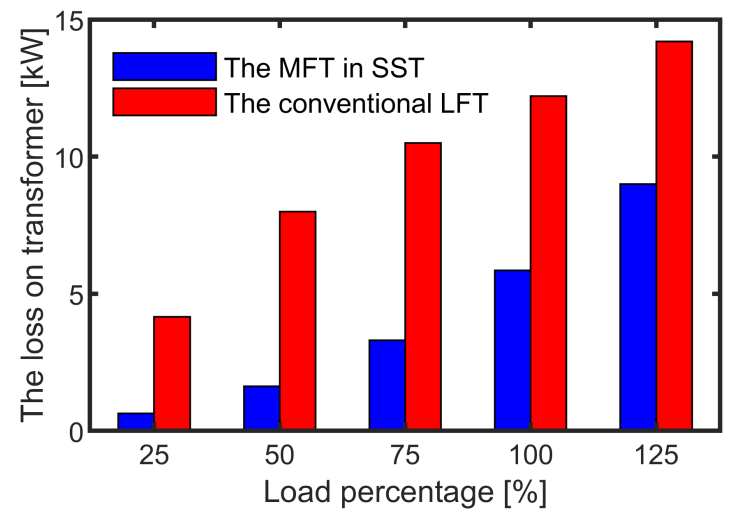

Fig. 15: The loss comparison between the conventional LFT and the SST MFT.

are modeled first. To compare the loss on transformer, five $200 \mathrm{~kW}$ arrays are connected to one LFT in the conventional system, while thirty SST arrays are providing 1MW to ac grid. For the simulated typical state-of-art LFT, the loss is higher than the MFT in the proposed SST system. At nominal condition, the loss in the MFT is $6.35 \mathrm{~kW}$ lower than the counterpart in the conventional LFT, for every $1 \mathrm{MW}$ section (Fig. 15). Based on the power losses on power semiconductor 


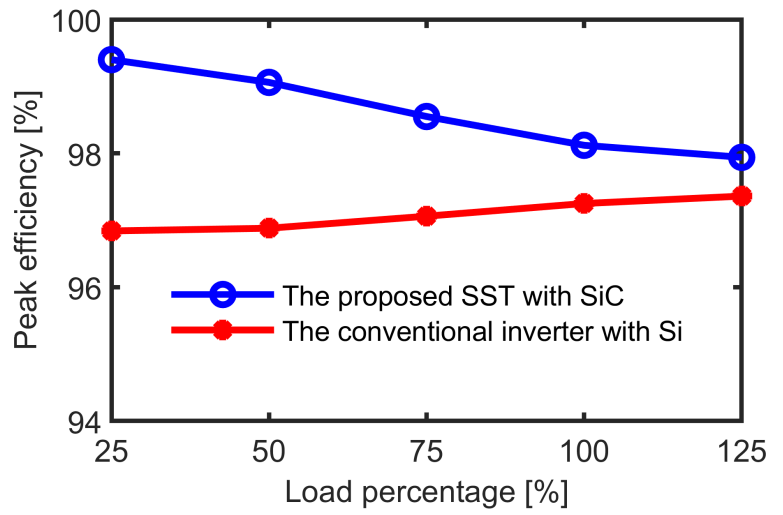

Fig. 16: The overall efficiency comparison between $\mathrm{Si}$ based conventional PV-BES system and the proposed $\mathrm{SiC}$ based solid state transformer PV-BES system.

devices, the total loss and overall efficiency is presented in Fig. 16. Since the power loss in the LFT is the relatively major portion of the total system losses, the efficiency follows the rating-efficiency trend of the transformer. For the SST system, the main part of the losses comes from the losses on the power semiconductor switches.

\section{CONCLUSION}

In this paper, a $\mathrm{SiC}$ based SST with power and voltage control is designed for utility-scale PV systems. With the proposed control design, maximum power point is extracted to maintain constant dc voltage for the PV system, and grid synchronized voltage/power is supplied to the power grid and the local load. When the solar energy is insufficient such as partial shading and intermittency, BES discharges to provide dc bus voltage compensation and power gap balancing. As a result, the proposed SST PV-BES system enhances the stability and reliability of the grid connection. Additionally, the simulation results validate the proposed control scheme. Moreover, power losses and efficiency analysis are investigated for the utility-scale PV farm. To further reduce power losses, $\mathrm{SiC}$ based SST are modeled and simulated. Although the traditional PV-BES system consists of only one inverter and there are three converters in the SST system, the total power losses with the example SiC SST system are still significantly lower than the traditional $\mathrm{Si}$ counterpart. At nominal load condition, the efficiency of the proposed SST system is $0.87 \%$ higher than the conventional Si based PV-battery system at the same condition. For low load condition such as $25 \%$, the proposed SiC SST system efficiency is $2.56 \%$ higher under the same condition. As a result, the proposed SiC based SST PV-BES system is more efficient.

\section{ACKNOWLEDGMENT}

The support of University of Kentucky, the L. Stanley Pigman endowment, and of ANSYS Inc. is gratefully acknowledged.

\section{REFERENCES}

[1] F. Blaabjerg and D. M. Ionel, "Renewable energy devices and systems with simulations in matlab ${ }^{\circledR}$ and ansys ${ }^{\circledR}, "$ in $C R C$
Press, Boca Raton, ISBN 9781498765824 - CAT K29112, 402p, 2017.

[2] S. Zengin and M. Boztepe, "Modified dual active bridge photovoltaic inverter for solid state transformer applications," in 2014 International Symposium on Fundamentals of Electrical Engineering (ISFEE), Nov 2014, pp. 1-4.

[3] T. Liu, Y. Xuan, X. Yang, P. Xu, Y. Li, L. Huang, and $\mathrm{X}$. Hao, "Adaptive voltage control scheme for dab based modular cascaded sst in pv application," in 2018 International Power Electronics Conference (IPEC-Niigata 2018 -ECCE Asia), May 2018, pp. 1478-1483.

[4] X. She, A. Q. Huang, and R. Burgos, "Review of solidstate transformer technologies and their application in power distribution systems," IEEE Journal of Emerging and Selected Topics in Power Electronics, vol. 1, no. 3, pp. 186-198, Sep. 2013.

[5] A. Q. Huang, Q. Zhu, L. Wang, and L. Zhang, "15 kv sic mosfet: An enabling technology for medium voltage solid state transformers," CPSS Transactions on Power Electronics and Applications, vol. 2, no. 2, pp. 118-130, 2017.

[6] A. Shojaei and G. Jos, "A topology for three-stage solid state transformer," in 2013 IEEE Power Energy Society General Meeting, July 2013, pp. 1-5.

[7] X. She, R. Burgos, G. Wang, F. Wang, and A. Q. Huang, "Review of solid state transformer in the distribution system: From components to field application," in 2012 IEEE Energy Conversion Congress and Exposition (ECCE), Sep. 2012, pp. 4077-4084.

[8] X. Ma, X. Yang, F. Zhang, L. Huang, Z. Li, and H. Song, "A control scheme of three phase solid state transformer for pv generation based on improved voltage-tracking method of dc links," in 2017 IEEE Applied Power Electronics Conference and Exposition (APEC), March 2017, pp. 474-479.

[9] C. Yeh and J. Lai, "A study on high frequency transformer design in medium-voltage solid-state transformers," in 2018 Asian Conference on Energy, Power and Transportation Electrification (ACEPT), Oct 2018, pp. 1-5.

[10] A. C. Nair and B. G. Fernandes, "A solid state transformer for integration of high power pv plant to medium voltage grid," in 2018 IEEE International Conference on Power Electronics, Drives and Energy Systems (PEDES), Dec 2018, pp. 1-6.

[11] J. Wang, B. Gu, Q. Duan, C. Ma, B. Ji, and J. You, "Control strategy of solid state power electronic transformer under voltage disturbance conditions," in IECON 2015 - 41st Annual Conference of the IEEE Industrial Electronics Society, Nov 2015, pp. $003081-003085$.

[12] T. Liu, X. Yang, W. Chen, Y. Li, Y. Xuan, L. Huang, and X. Hao, "Design and implementation of high efficiency control scheme of dual active bridge based $10 \mathrm{kv} / 1 \mathrm{mw}$ solid state transformer for pv application," IEEE Transactions on Power Electronics, vol. 34, no. 5, pp. 4223-4238, May 2019.

[13] V. Rallabandi, O. M. Akeyo, N. Jewell, and D. M. Ionel, "Incorporating battery energy storage systems into multi-mw grid connected pv systems," IEEE Transactions on Industry Applications, vol. 55, no. 1, pp. 638-647, Jan 2019.

[14] “ANSYS Inc." 2019, www.ansys.com.

[15] "E. W. Brown solar facility live solar generation data," 2018, lge-ku.com/live-solar-generation.

[16] "Infineon FF1200R12KE3," 2019, www.infineon.com.

[17] "Wolfspeed CAS300M12BM2," 2019, www.wolfspeed.com.

[18] "EATON Cooper Power," 2019, www.eaton.com.

[19] D. Ruiz-Robles, V. Venegas-Rebollar, A. Anaya-Ruiz, E. Moreno-Goytia, and J. Rodríguez-Rodríguez, "Design and prototyping medium-frequency transformers featuring a nanocrystalline core for dc-dc converters," Energies, vol. 11, no. 8, p. 2081, 2018. 